\title{
Analysis of the mitochondrial subproteome of the human cell line AGE1.HN - a contribution to a systems biology approach
}

\author{
Eva Schräder ${ }^{1 *}$, Sebastian Scholz ${ }^{1}$, Volker Sandig ${ }^{2}$, Raimund Hoffrogge ${ }^{1}$, Thomas Noll ${ }^{1}$ \\ From 22nd European Society for Animal Cell Technology (ESACT) Meeting on Cell Based Technologies \\ Vienna, Austria. 15-18 May 2011
}

\section{Background}

In Systems Biology approaches a good amount of reliable data is essential for effective modelling of distinct biological processes. Hence the focus of the SysLogicsProject was on modelling of the central metabolism using different functional genomic techniques. For displaying the involved proteins, it is crucial to enrich them using subproteomic fractions. As many enzymes being involved in central metabolism are located in the mitochondria, we investigated the expression dynamics of mitochondrial proteins during batch cultivations of human AGE1.HN cells (ProBioGen, Berlin, Germany).

\section{Materials and methods}

Cultivation and isolation of mitochondria

Cultivations were performed as batch-processes with chemically-defined and animal-component-free media 42-MAX-UB (Teutocell, Bielefeld, Germany) with addition of $5 \mathrm{mM}$ glutamine. The processes were performed in a $20 \mathrm{~L}$-stainless steel vessel (Sartorius-Stedim, Germany). After disintegration of cells by addition of glass beads $(0,1 \mathrm{~mm}$ diameter) and vortexing, the isolation of mitochondria was performed with sucrose density-centrifugation in an ultracentrifuge (Beckman Coulter, Krefeld, Germany). Protein extraction from mitochondria was performed with lysis-buffer, which contains Tris$\mathrm{HCl}, \mathrm{NaCl}, \mathrm{EDTA}$, SDS, PMSF and NP40.

\section{Proteomics}

The first dimension of 2D-electrophoresis was performed with Ettan IPGPhor3-system*, using pH 3-11

\footnotetext{
* Correspondence: esc@zellkult.techfak.uni-bielefeld.de

${ }^{1}$ Institute for Cell Culture Technology, University of Bielefeld, Bielefeld, Germany

Full list of author information is available at the end of the article
}

(NL) IPG-strips*. For generating the mitochondrial master-map, six gels with $0.45 \mathrm{mg}$ protein extract were prepared. For DIGE-approaches $0.15 \mathrm{mg}$ were applied, each sample with four replicates. Second dimension was accomplished with Ettan Dalt six Electrophoresis System*. 2DE-gels with DIGE-staining were processed with Ettan Dige Imager*. Software evaluation was implemented with Delta2D (Decodon, Germany).

After isolation and tryptic digest protein-identification was performed with MS/MS using MALDI-ToF/ToF (UltrafleXtreme, Bruker, Germany), followed by database-searching.

*(GE Healthcare, Sweden)

\section{Results}

For proteomic analyses, mitochondria were isolated and successfully confirmed by staining with Rhodamine 123, a mitochondria-specific fluorescent dye. 2DE-gels of mitochondrial fraction led to 519 separated spots. 280 proteins could be reliably identified, out of which more than $50 \%$ are exclusively mitochondrial. This $2 \mathrm{D}$-proteome map is our basis for approaches of analysing different protein expression in mitochondria.

Two standardised batch cultivations in a $20 \mathrm{~L}$-stirred tank stainless steel vessel were carried out with daily sampling for metabolomics, transcriptomics, metabolic flux and proteomics, in order to generate reliable and comparable samples. Batch cultivation in $20 \mathrm{~L}$-scale of AGE1.HN.AAT shows cell densities and duration as expected. Glucose- and lactate-concentrations developed also as estimated in standard batch culture (see fig. 1a+b).

Subproteomic DIGE-analysis of mitochondrial proteins in those $20 \mathrm{~L}$-batch cultivations resulted in 114 proteins that were significantly differently expressed in the first and 206 proteins in the second process during 

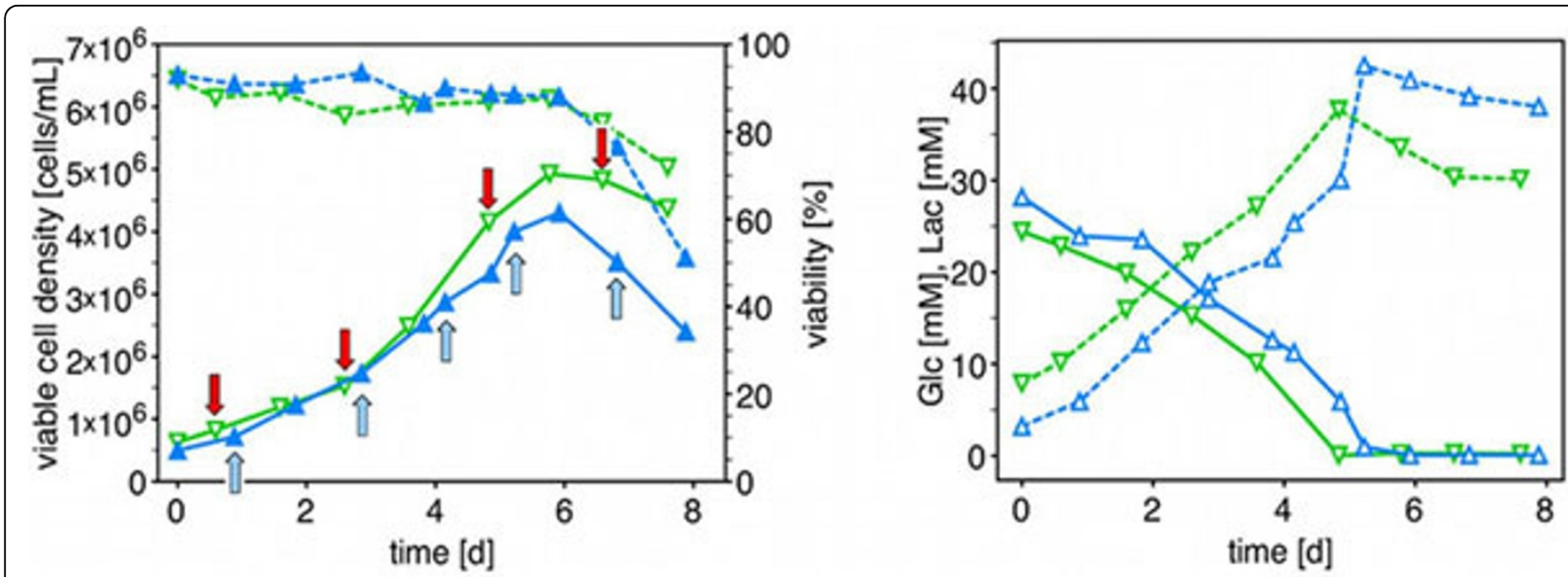

Figure 1 a (left): VCD (solid) and viability (dashed) during batch process 1 (open squares) and 2 (closed triangles) Fig. 1b (right): Concentrations of glucose (solid) and lactate (dashed) during batch process 1 (open squares) and 2 (closed triangles).

Table 1 Examples of expression profiles of TCA and respiratory chain enzymes. Expression normalised on sample point 1.

\begin{tabular}{llllllll}
\hline & ZE1 $(2,6 \mathrm{~d})$ & ZE1 $(4,8 \mathrm{~d})$ & ZE1 $(6,5 \mathrm{~d})$ & ZE2 (2,9 d) & ZE2 $(4,2 \mathrm{~d})$ & ZE2 (5,3 d) & ZE2 (6,9 d) \\
\hline Pyruvate dehydrogenase E1a & 1,53 & 1,6 & 0,83 & 1,51 & 1,56 & 1,09 & 0,87 \\
L-lactate dehydrogenase B chain & 0,95 & 0,87 & 5,18 & 0,98 & 1,53 & 2,4 & 3,26 \\
Dihydrolipoamide S-succinyltransferase & 2,37 & 2,18 & 1,2 & 2,06 & 1,92 & 1,73 & 0,89 \\
Electron transfer flavoprotein alpha & 2,57 & 2,24 & 1,38 & 2,29 & 1,88 & 1,99 & 1,45 \\
\hline
\end{tabular}

cultivation time ( $\mathrm{p}<0.05$, factorial Anova). More than 40 proteins were found to be significantly regulated in both experiments. DIGE-approaches of mitochondrial fraction of both batch cultivations led to expression profiles of identified proteins. Four examples of expression profiles of typical proteins of TCA and respiratory chain are shown below in table 1.

Apart from these examples it was possible to observe important biological processes with our 2DE-approach, as for instance:

- tricarboxylic acid cycle

- respiratory chain

- anti-apoptosis and apoptosis

- signal transduction

- chaperone/protein folding and processing

- fatty acid-/lipid-metabolism

- amino acid-degradation

- inhibition of DNA-synthesis

- ketone-metabolism

- protein-biosynthesis

- redox-regulation

- ubiquinone-biosynthesis

Hence, we see this data as a valuable contribution to the Systems Biology approach for modelling central metabolism events.

\section{Conclusions}

Reproducible methods for isolation of mitochondrial proteins from cultured cells for further analysis, such as proteomic 2DE-approaches, have been established. DIGE-analysis of mitochondrial subproteome of AGE1. HN.AAT cells result in the identification of more than 280 identified proteins, of which more than 40 are regulated during batch cultivation. Proteomics data in conjunction with the other "omics"-results offer a promising basis for characterisation of central metabolism during Systems Biology approaches.

\section{Acknowledgments}

This work is part of the SysLogics Project: Systems biology of cell culture for biologics, founded by German Ministry for Education and Research (BMBF).

\section{Author details}

'Institute for Cell Culture Technology, University of Bielefeld, Bielefeld, Germany. ${ }^{2}$ ProBioGen AG, Berlin, Germany.

Published: 22 November 2011

doi:10.1186/1753-6561-5-S8-P86

Cite this article as: Schräder et al: Analysis of the mitochondrial subproteome of the human cell line AGE1.HN - a contribution to a systems biology approach. BMC Proceedings 2011 5(Suppl 8):P86. 